Mathematical Modelling And Analysis

Volume 20 Number 3, May 2015, 409-421

http://dx.doi.org/10.3846/13926292.2015.1049970

(c) Vilnius Gediminas Technical University, 2015
Publisher: Taylor\&Francis and VGTU

http://www.tandfonline.com/TMMA

ISSN: $1392-6292$

eISSN: 1648-3510

\title{
Expert Estimates Averaging by Constructing Intuitionistic Fuzzy Triangles
}

\author{
${ }^{a}$ Mykolas Romeris University \\ Ateities 20, LT-08303 Vilnius, Lihuania \\ ${ }^{b}$ Vilnius Gediminas Technical University \\ Sauletekio 11, LT-10223 Vilnius, Lihuania \\ E-mail: krylovas@mruni.eu; akr@vgtu.lt \\ E-mail(corresp.): Natalja.Kosareva@vgtu.lt
}

Aleksandras Krylovas ${ }^{a, b}$ and Natalja Kosareva ${ }^{b}$

Received September 22, 2012; revised March 7, 2015; published online May 15, 2015

\begin{abstract}
The problem of ranking (sorting) of $m$ alternatives is considered when experts evaluate each alternative according to $k$ criteria. Functions of arithmetic and geometric averages are constructed for decision making. We present a generalization of this scheme when there are evaluation matrices of several experts and this information is aggregated in the form of triangular intuitionistic fuzzy numbers. Fuzzy triangles were constructed with different uncertainty levels, experts decision matrices and the number of experts varied from 2 to 5 . Moreover, method for construction of experts decision probability matrices is proposed in the paper. Ranking results obtained by performing Monte Carlo simulations. Probabilities of errors are compared for arithmetic, geometric, fuzzy arithmetic and fuzzy geometric averages.

Keywords: intuitionistic fuzzy numbers, Monte Carlo simulations, multiple criteria decision making.
\end{abstract}

AMS Subject Classification: 90B90; 62P25; $62 \mathrm{C} 86$.

\section{Introduction}

Fuzzy sets theory which has become classic since its appearance in the works of L.A. Zadeh is generally acknowledged as an effective tool for decision-making models [3]. Intuitionistic fuzzy sets offered by K. Atanassov [2] are the generalization of the classic fuzzy sets widely used in mathematical modelling. We mention just a few recent publications, devoted to application of intuitionistic fuzzy sets in decision-making models: $[1,7,8]$. Separately we mention the papers where weighted averages of intuitionistic fuzzy numbers are used for decision making: $[5,16]$. Group decision-making methods based on some Hamacher aggregation operators are analyzed in [10]. Multiple attribute decision making problems with uncertain weights in intuitionistic fuzzy setting are investigated in [12]. Generalization of Multiple Criteria Decision Making (MCDM) - dealing 
with intuitionistic fuzzy numbers instead of crisp numbers - is often used for searching the best alternative in many fields of human activities. It was shown in [9] that in many cases intuitionistic fuzzy approach lets to obtain better results in solving Multiple Criteria Decision Making problems than dealing with real numbers and fuzzy numbers. The papers $[15,17]$ analyze application of triangular and trapezoidal intuitionistic fuzzy numbers for decision making. A new class of generalized intuitionistic fuzzy aggregation operators is developed in [6]. With respect to multiple attribute group decision making (MAGDM) problems in which both the attribute weights and the expert weights take the form of crisp numbers, new intuitionistic uncertain linguistic Heronian mean operators are proposed in [11]. Hesitant fuzzy multiple attribute decision making with incomplete weight information is investigated in [14]. Most often aggregation operators, which are proposed in the latest articles, are modifications of arithmetic and geometric averages. So it is important to compare precision of obtained solutions of these operators.

Theoretical aspect of the investigation deals with the justification of the correctness of mathematical operations, carried out with the fuzzy numbers, and practical aspects - case study calculations - are concerned with the a priori fuzzy variables. However, in practice these quantities are certain expert evaluations, whose expression in terms of fuzzy numbers (fuzzyfication) can be made in various ways $[13,17]$ and it can have a big impact on the ranking of alternatives. This problem was mentioned in [9]. The aim of this paper is to offer algorithm of construction intuitionistic fuzzy triangles when each alternative is assessed separately according to each criteria by $n$ experts. Evaluations are certain grades which may contain natural interpretation in linguistic fuzzy notions: project fully complies with the requirements, partly meets the requirements, etc. We offer the original method for construction of fuzzy triangles with respect to all experts' estimates evaluating the commonness and diversity of their opinions.

It should be remembered that intuitionistic fuzzy set $S=\{\langle x, \mu(x), \nu(x)\rangle$, $x \in E\}$ is characterized by two functions $\mu: E \rightarrow[0,1]$ and $\nu: E \rightarrow[0,1]$, satisfying the condition $0 \leqslant \mu(x)+\nu(x) \leqslant 1$. Function $\mu$ describes the degree of belonging of the element to the fuzzy set $S$, and function $\nu$ - the degree of not belonging. K. Atanassov [2] submitted such an interpretation of these features: $\mu(x)$ simulates a certain degree of national government support, $\nu(x)-$ a degree of opposition. Further we based on intuitionistic triangular fuzzy number definition proposed in [17]. In this case when all the experts evaluate the same project equally, the function $\nu(x)$ acquires the minimum value. If some expert opinions vary - function $\nu(x)$ value is greater.

\section{Modeling of Expert's Assessments Results by Triangu- lar Intuitionistic Fuzzy Numbers}

Suppose, that $n$ experts evaluate projects according to certain criteria (attributes): $t_{i} \in\{1,2, \ldots, B\}, i=1,2, \ldots, m$. It means that each criterion measured on a scale from 1 point to $B$ points. Let us denote $\alpha, \beta \in(0,1)$ the numbers describing measures of uncertainty: $\alpha$ - degree of membership and 
$\beta$ - degree of non-membership of triangular intuitionistic fuzzy numbers. Our purpose is to construct project's estimates in the form of triangular intuitionistic fuzzy numbers: $T=\left(\left[a^{L}, a^{M}, a^{U}\right],\left[b^{L}, b^{M}, b^{U}\right]\right)$, i.e. numbers should satisfy the inequalities:

$$
0 \leqslant a^{L} \leqslant a^{M} \leqslant a^{U} \leqslant 1, \quad 0 \leqslant b^{L} \leqslant b^{M} \leqslant b^{U} \leqslant 1, \quad a^{U}+b^{U} \leqslant 1 .
$$

Let us denote $a_{B, n, \alpha}^{j}, b_{B, n, \beta}^{j} \in(0 ; 1), j=L, M, U$ the numbers depending on all $n$ experts estimates and satisfying the inequalities:

$$
a_{B, n, \alpha}^{L} \leqslant a_{B, n, \alpha}^{M} \leqslant a_{B, n, \alpha}^{U}, \quad b_{B, n, \beta}^{L} \leqslant b_{B, n, \beta}^{M} \leqslant b_{B, n, \beta}^{U} .
$$

Next, let us denote

$$
d=\min _{t_{1}, t_{2}, \ldots, t_{m}}\left\{a_{B, n, \alpha}^{L}, b_{B, n, \beta}^{L}\right\}, \quad D=\max _{t_{1}, t_{2}, \ldots, t_{m}}\left\{a_{B, n, \alpha}^{U}+b_{B, n, \beta}^{U}\right\}
$$

and consider the normalizing transformations:

$$
a_{j}=\frac{a_{B, n, \alpha}^{j}-d}{D-d}, \quad b_{j}=\frac{b_{B, n, \alpha}^{j}-d}{D-d}, \quad j=L, M, U .
$$

The numbers obtained after normalizing transformations (2.2) satisfy conditions (2.1) for the components of triangular intuitionistic fuzzy numbers. When constructing intuitionistic fuzzy numbers $T$, in which information gathered from all experts is aggregated, we are following methodological principles. Triple $\left[a_{B, n, \alpha}^{L}, a_{B, n, \alpha}^{M}, a_{B, n, \alpha}^{U}\right]$ is treated as triangular fuzzy number where value $a_{B, n, \alpha}^{M}$ is determined according to the majority's opinion. Numbers $a_{B, n, \alpha}^{L}$ and $a_{B, n, \alpha}^{U}$ are constructed according to extreme opinions of the experts. Triple $\left[b_{B, n, \alpha}^{L}, b_{B, n, \alpha}^{M}, b_{B, n, \alpha}^{U}\right]$ is treated as uncertainty in measurements of experts assessments. Value $b_{B, n, \alpha}^{M}$ is higher with the greater diversity of experts opinions. We offer algorithm for construction of numbers $a^{j}, b^{j}$ and we are looking for parameters $\alpha$ and $\beta$ values maximizing its effectiveness. Such method of ranking alternatives can be particularly effective when the number of experts $n$ is not large.

Hereinafter we will consider special case when $B=3$, i.e. each expert evaluates project's components (attributes) with 3 values: does not satisfy certain criterion $\left(t_{i}=1\right)$, partly satisfies certain criterion $\left(t_{i}=2\right)$ and satisfies certain criterion $\left(t_{i}=3\right)$.

Let us denote $n_{j}, j=1,2,3,\left(n_{1}+n_{2}+n_{3}=n\right)$ - number of experts evaluated certain project's attribute by estimate $j$. Then the collection $\left(n_{1}, n_{2}, n_{3}\right)$ shows how experts estimated this project's attribute. For example, in the case of 3 experts, the collection $(0,3,0)$ shows that opinions of all 3 experts were "project partly satisfies certain criterion".

In Table 1 triangular intuitionistic fuzzy numbers are constructed from the separate experts' opinions for all possible situations in the cases of 2 experts $(n=2)$ and 3 experts $(n=3)$.

Likewise such generalized estimates in the form of triangular IFNs could be constructed for the higher number of experts. After the construction of 
Table 1. Generalized estimates as the components of triangular intuitionistic fuzzy numbers, calculated for the cases of 2 and 3 experts $(n=2,3)$.

\begin{tabular}{ccc}
\hline$\left(n_{1}, n_{2}, n_{3}\right)$ & {$\left[a_{3,2, \alpha}^{L}, a_{3,2, \alpha}^{M}, a_{3,2, \alpha}^{U}\right]$} & {$\left[b_{3,2, \beta}^{L}, b_{3,2, \beta}^{M}, b_{3,2, \beta}^{U}\right]$} \\
\hline$(2,0,0)$ & $(1-2 \alpha, 1,1+2 \alpha)$ & $(0, \beta, 2 \beta)$ \\
$(1,1,0)$ & $(1-\alpha, 3 / 2,2+\alpha)$ & $(\beta, 2 \beta, 3 \beta)$ \\
$(1,0,1)$ & $(1-\alpha, 2,3+\alpha)$ & $(2 \beta, 3 \beta, 4 \beta)$ \\
$(0,2,0)$ & $(2-2 \alpha, 2,2+2 \alpha)$ & $(0, \beta, 2 \beta)$ \\
$(0,1,1)$ & $(2-\alpha, 5 / 2,3+\alpha)$ & $(\beta, 2 \beta, 3 \beta)$ \\
$(0,0,2)$ & $(3-2 \alpha, 3,3+2 \alpha)$ & $(0, \beta, 2 \beta)$ \\
\hline$\left(n_{1}, n_{2}, n_{3}\right)$ & {$\left[a_{3,3, \alpha}^{L}, a_{3,3, \alpha}^{M}, a_{3,3, \alpha}^{U}\right]$} & {$\left[b_{3,3, \beta}^{L}, b_{3,3, \beta}^{M}, b_{3,3, \beta}^{U}\right]$} \\
$(3,0,0)$ & $(1-3 \alpha, 1,1+3 \alpha)$ & $(0, \beta, 2 \beta)$ \\
$(2,1,0)$ & $(1-2 \alpha, 1,2+\alpha)$ & $(2 \beta, 2 \beta, 3 \beta)$ \\
$(2,0,1)$ & $(1-2 \alpha, 1,3+\alpha)$ & $(2 \beta, 3 \beta, 4 \beta)$ \\
$(1,1,1)$ & $(1-\alpha, 2,1+\alpha)$ & $(\beta, 2 \beta, 3 \beta)$ \\
$(0,2,1)$ & $(2-2 \alpha, 2,3+\alpha)$ & $(0, \beta, 2 \beta)$ \\
$(0,3,0)$ & $(2-3 \alpha, 2,2+3 \alpha)$ & $(\beta, 2 \beta, 3 \beta)$ \\
$(0,1,2)$ & $(2-\alpha, 3,3+2 \alpha)$ & $(\beta, 3 \beta, 4 \beta)$ \\
$(1,0,2)$ & $(1-\alpha, 3,3+2 \alpha)$ & $(0, \beta, 2 \beta)$ \\
$(1,2,0)$ & $(1-\alpha, 2,2+2 \alpha)$ & \\
$(0,0,3)$ & $(3-3 \alpha, 3,3+3 \alpha)$ & \\
\hline
\end{tabular}

generalized estimates, they are normalized by formulas (2.2). Then weighted averaging operator's values are calculated for each project for consolidation of expert's evaluations according to each criteria, calculation of each project's score and ranking alternatives.

\section{Simulation of Expert Assessments}

Suppose, that the true value of project's certain component (attribute) $t \in$ $\{1,2,3\}$ is known. Let us denote by $p_{t j}$ conditional probability, that expert's estimate is equal to $j \in\{1,2,3\}$ provided that true value is equal to $t$. It's natural to require: $(\forall t \neq j) p_{t t}>p_{t j}$. This condition means that expert must be qualified and make correct assessment more often than incorrect. All values $p_{i j}$ construct matrix $P=\left(\begin{array}{lll}p_{11} & p_{12} & p_{13} \\ p_{21} & p_{22} & p_{23} \\ p_{31} & p_{32} & p_{33}\end{array}\right)$. Let us say, that expert must make incorrect and correct decisions symmetrically, i.e. $p_{12}=p_{32}, p_{13}=p_{31}, p_{21}=p_{23}$. For example, if we choose matrix $P_{1}=\left(\begin{array}{ccc}0.7 & 0.25 & 0.05 \\ 0.25 & 0.5 & 0.25 \\ 0.05 & 0.25 & 0.7\end{array}\right)$, then experts' estimates $\left(n_{1}, n_{2}, n_{3}\right)$ of an attribute could be simulated with conditional probability distributions calculated in Table 2 .

\section{Determining Experts Decision Matrix}

The paper [4] deals with managerial task when assessments of criteria $x_{1}, x_{2}$, $\ldots, x_{6}$ made by 22 experts are provided. Table 3 shows fragment of the data matrix. The higher score corresponds to the higher priority of criteria. It 
Table 2. Conditional probability distributions of the attribute estimates when probability matrix is $P_{1}$ and $n=2$.

\begin{tabular}{lccc}
\hline$\left(n_{1}, n_{2}, n_{3}\right)$ & $t=1$ & $t=2$ & $t=3$ \\
\hline$(2,0,0)$ & $p_{11}^{2}=0.49$ & $p_{21}^{2}=0.0625$ & $p_{31}^{2}=0.0025$ \\
$(1,1,0)$ & $2 p_{11} p_{12}=0.35$ & $2 p_{21} p_{22}=0.25$ & $2 p_{31} p_{32}=0.025$ \\
$(1,0,1)$ & $2 p_{11} p_{13}=0.07$ & $2 p_{21} p_{23}=0.125$ & $2 p_{31} p_{33}=0.0625$ \\
$(0,2,0)$ & $p_{12}^{2}=0.0625$ & $p_{22}^{2}=0.25$ & $p_{32}^{2}=0.07$ \\
$(0,1,1)$ & $2 p_{12} p_{13}=0.025$ & $2 p_{22} p_{23}=0.25$ & $2 p_{32} p_{33}=0.35$ \\
$(0,0,2)$ & $p_{13}^{2}=0.0025$ & $p_{22}^{2}=0.0625$ & $p_{33}^{2}=0.49$ \\
\hline
\end{tabular}

Table 3. Evaluation of criteria $x_{1}-x_{6}$ priority by 22 experts.

\begin{tabular}{ccccccc}
\hline Expert & $x_{1}$ & $x_{2}$ & $x_{3}$ & $x_{4}$ & $x_{5}$ & $x_{6}$ \\
\hline 1 & 6 & 1 & 5 & 3 & 2 & 4 \\
$\ldots$ & $\ldots$ & $\ldots$ & $\ldots$ & $\ldots$ & $\ldots$ & $\ldots$ \\
22 & 4 & 1 & 6 & 3 & 2 & 5 \\
\hline$\sum_{\text {rank }}$ & 114 & 41 & 97 & 42 & 96 & 72 \\
\hline
\end{tabular}

means that the first expert ranked attributes in this order:

$$
x_{1} \succ x_{3} \succ x_{6} \succ x_{4} \succ x_{5} \succ x_{2}
$$

and the 22-nd expert - in this order:

$$
x_{3} \succ x_{6} \succ x_{1} \succ x_{4} \succ x_{5} \succ x_{2} .
$$

The calculated sums of column ranks allow setting some "average" expert opinion which we will assume as "true" (the lower rank corresponds to the higher priority):

$$
x_{1} \succ x_{3} \succ x_{5} \succ x_{6} \succ x_{4} \succ x_{2} .
$$

We will use this information to estimate expert assessments probability matrix. Suppose that significance of each criterion $x_{i}$ should be described in a single attribute: $\mathrm{S}$ - significant, $\mathrm{A}$ - the average, I - irrelevant. Select triplets of variable $X=\left(x_{1}, x_{2}, x_{3}, x_{4}, x_{5}, x_{6}\right)$ components and write the first expert's preferences (4.1), the "true" preferences (4.3) and matrices of attributes conferment. For example, let us take the variables $\left\{x_{3}, x_{4}, x_{5}\right\}$. Then, the first expert and "true" preferences recorded as follows: $x_{3} \succ x_{4} \succ x_{5}$ and $x_{3} \succ x_{5} \succ x_{4}$. The same information could be written by the substitution: $\left(\begin{array}{ccc}S & A & I \\ S & I & A\end{array}\right)$. Either fill in the table cells so that the rows correspond to the first expert assigned attributes and columns - true attributes:

\begin{tabular}{c|c|c|c} 
& $S$ & $A$ & $I$ \\
\hline$S$ & 1 & 0 & 0 \\
\hline$A$ & 0 & 0 & 1 \\
\hline$I$ & 0 & 1 & 0 \\
\hline
\end{tabular}


Accordingly, the position of the unit in the first row of matrix means that the attribute $S$ was provided fairly ("true" attribute is also $S$ ), the unit position in the second row means that the attribute A was granted instead of the "true" attribute $I$. Note that all possible cases correspond to the 6 different arrays:

$$
\begin{array}{ll}
E_{123}=\left(\begin{array}{lll}
1 & 0 & 0 \\
0 & 1 & 0 \\
0 & 0 & 1
\end{array}\right), & E_{132}=\left(\begin{array}{lll}
1 & 0 & 0 \\
0 & 0 & 1 \\
0 & 1 & 0
\end{array}\right), \quad E_{213}=\left(\begin{array}{lll}
0 & 1 & 0 \\
1 & 0 & 0 \\
0 & 0 & 1
\end{array}\right), \\
E_{231}=\left(\begin{array}{lll}
0 & 1 & 0 \\
0 & 0 & 1 \\
1 & 0 & 0
\end{array}\right), & E_{312}=\left(\begin{array}{lll}
0 & 0 & 1 \\
1 & 0 & 0 \\
0 & 1 & 0
\end{array}\right), \quad E_{321}=\left(\begin{array}{lll}
0 & 0 & 1 \\
0 & 1 & 0 \\
1 & 0 & 0
\end{array}\right) .
\end{array}
$$

In Table 4 all 20 possible choices for preferences of the three variables of 6 variables $x_{1}-x_{6}$ made by first expert are presented.

\begin{tabular}{|c|c|c|c|c|}
\hline Nr. & Triplet & Preferences & Attributes & Matrix \\
\hline 1 & $\left\{x_{1}, x_{2}, x_{3}\right\}$ & $\begin{array}{l}x_{1} \succ x_{3} \succ x_{2} \\
x_{1} \succ x_{3} \succ x_{2}\end{array}$ & $\left(\begin{array}{lll}S & A & I \\
S & A & I\end{array}\right)$ & $E_{123}$ \\
\hline 2 & $\left\{x_{1}, x_{2}, x_{4}\right\}$ & $\begin{array}{l}x_{1} \succ x_{4} \succ x_{2} \\
x_{1} \succ x_{4} \succ x_{2}\end{array}$ & $\left(\begin{array}{lll}S & A & I \\
S & A & I\end{array}\right)$ & $E_{123}$ \\
\hline 3 & $\left\{x_{1}, x_{2}, x_{5}\right\}$ & $\begin{array}{l}x_{1} \succ x_{5} \succ x_{2} \\
x_{1} \succ x_{5} \succ x_{2}\end{array}$ & $\left(\begin{array}{lll}S & A & I \\
S & A & I\end{array}\right)$ & $E_{123}$ \\
\hline 4 & $\left\{x_{1}, x_{2}, x_{6}\right\}$ & $\begin{array}{l}x_{1} \succ x_{6} \succ x_{2} \\
x_{1} \succ x_{6} \succ x_{2}\end{array}$ & $\left(\begin{array}{lll}S & A & I \\
S & A & I\end{array}\right)$ & $E_{123}$ \\
\hline 5 & $\left\{x_{1}, x_{3}, x_{4}\right\}$ & $\begin{array}{l}x_{1} \succ x_{3} \succ x_{4} \\
x_{1} \succ x_{3} \succ x_{4}\end{array}$ & $\left(\begin{array}{lll}S & A & I \\
S & A & I\end{array}\right)$ & $E_{123}$ \\
\hline 6 & $\left\{x_{1}, x_{3}, x_{5}\right\}$ & $\begin{array}{l}x_{1} \succ x_{3} \succ x_{5} \\
x_{1} \succ x_{3} \succ x_{5} \\
\end{array}$ & $\left(\begin{array}{lll}S & A & I \\
S & A & I\end{array}\right)$ & $E_{123}$ \\
\hline 7 & $\left\{x_{1}, x_{3}, x_{6}\right\}$ & $\begin{array}{l}x_{1} \succ x_{3} \succ x_{6} \\
x_{1} \succ x_{3} \succ x_{6} \\
\end{array}$ & $\left(\begin{array}{lll}S & A & I \\
S & A & I\end{array}\right)$ & $E_{123}$ \\
\hline 8 & $\left\{x_{1}, x_{4}, x_{5}\right\}$ & $\begin{array}{l}x_{1} \succ x_{4} \succ x_{5} \\
x_{1} \succ x_{5} \succ x_{4}\end{array}$ & $\left(\begin{array}{lll}S & A & I \\
S & I & A\end{array}\right)$ & $E_{132}$ \\
\hline 9 & $\left\{x_{1}, x_{4}, x_{6}\right\}$ & $\begin{array}{l}x_{1} \succ x_{6} \succ x_{4} \\
x_{1} \succ x_{6} \succ x_{4} \\
\end{array}$ & $\left(\begin{array}{lll}S & A & I \\
S & A & I\end{array}\right)$ & $E_{123}$ \\
\hline 10 & $\left\{x_{1}, x_{5}, x_{6}\right\}$ & $\begin{array}{l}x_{1} \succ x_{6} \succ x_{5} \\
x_{1} \succ x_{5} \succ x_{6}\end{array}$ & $\left(\begin{array}{lll}S & A & I \\
S & I & A\end{array}\right)$ & $E_{132}$ \\
\hline 11 & $\left\{x_{2}, x_{3}, x_{4}\right\}$ & $\begin{array}{l}x_{3} \succ x_{4} \succ x_{2} \\
x_{3} \succ x_{4} \succ x_{2} \\
\end{array}$ & $\left(\begin{array}{lll}S & A & I \\
S & A & I\end{array}\right)$ & $E_{123}$ \\
\hline 12 & $\left\{x_{2}, x_{3}, x_{5}\right\}$ & $\begin{array}{l}x_{3} \succ x_{5} \succ x_{2} \\
x_{3} \succ x_{5} \succ x_{2} \\
\end{array}$ & $\left(\begin{array}{lll}S & A & I \\
S & A & I\end{array}\right)$ & $E_{123}$ \\
\hline 13 & $\left\{x_{2}, x_{3}, x_{6}\right\}$ & $\begin{array}{l}x_{3} \succ x_{6} \succ x_{2} \\
x_{3} \succ x_{6} \succ x_{2} \\
\end{array}$ & $\left(\begin{array}{lll}S & A & I \\
S & A & I\end{array}\right)$ & $E_{123}$ \\
\hline 14 & $\left\{x_{2}, x_{4}, x_{5}\right\}$ & $\begin{array}{l}x_{4} \succ x_{5} \succ x_{2} \\
x_{5} \succ x_{4} \succ x_{2} \\
\end{array}$ & $\left(\begin{array}{lll}S & A & I \\
A & S & I\end{array}\right)$ & $E_{213}$ \\
\hline 15 & $\left\{x_{2}, x_{4}, x_{6}\right\}$ & $\begin{array}{l}x_{6} \succ x_{4} \succ x_{2} \\
x_{6} \succ x_{4} \succ x_{2}\end{array}$ & $\left(\begin{array}{lll}S & A & I \\
S & A & I\end{array}\right)$ & $E_{123}$ \\
\hline 16 & $\left\{x_{2}, x_{5}, x_{6}\right\}$ & $\begin{array}{l}x_{6} \succ x_{5} \succ x_{2} \\
x_{5} \succ x_{6} \succ x_{2}\end{array}$ & $\left(\begin{array}{lll}S & A & I \\
A & S & I\end{array}\right)$ & $E_{213}$ \\
\hline 17 & $\left\{x_{3}, x_{4}, x_{5}\right\}$ & $\begin{array}{l}x_{3} \succ x_{4} \succ x_{5} \\
x_{3} \succ x_{5} \succ x_{4} \\
\end{array}$ & $\left(\begin{array}{lll}S & A & I \\
S & I & A\end{array}\right)$ & $E_{132}$ \\
\hline 18 & $\left\{x_{3}, x_{4}, x_{6}\right\}$ & $\begin{array}{l}x_{3} \succ x_{6} \succ x_{4} \\
x_{3} \succ x_{6} \succ x_{4} \\
\end{array}$ & $\left(\begin{array}{lll}S & A & I \\
S & A & I\end{array}\right)$ & $E_{123}$ \\
\hline 19 & $\left\{x_{3}, x_{5}, x_{6}\right\}$ & $\begin{array}{l}x_{3} \succ x_{6} \succ x_{5} \\
x_{3} \succ x_{5} \succ x_{6}\end{array}$ & 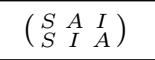 & $E_{132}$ \\
\hline 20 & $\left\{x_{4}, x_{5}, x_{6}\right\}$ & $\begin{array}{l}x_{6} \succ x_{4} \succ x_{5} \\
x_{5} \succ x_{6} \succ x_{4}\end{array}$ & $\left(\begin{array}{ccc}S & A & I \\
A & I & S\end{array}\right)$ & $E_{231}$ \\
\hline
\end{tabular}

Table 4. 20 possible choices of 3 variables from $x_{1}-x_{6}$ with first expert evaluations. 
So, frequencies from Table 4 are sorted and summarized in the Table 5 .

Table 5. Summarized frequencies of the first expert.

\begin{tabular}{cccccccc}
\hline & $E_{123}$ & $E_{132}$ & $E_{213}$ & $E_{231}$ & $E_{312}$ & $E_{321}$ & $\Sigma$ \\
\hline$n_{i j k}$ & 13 & 4 & 1 & 2 & 0 & 0 & 20 \\
\hline
\end{tabular}

Elements of the first expert probability matrix are calculated as the average values of discrete random variables $\left\{E_{i j k}, \frac{n_{i j k}}{20}\right\}$ :

$$
\begin{aligned}
P^{(1)}= & \left(\begin{array}{ccc}
p_{11}^{(1)} & p_{12}^{(1)} & p_{13}^{(1)} \\
p_{21}^{(1)} & p_{22}^{(1)} & p_{23}^{(1)} \\
p_{31}^{(1)} & p_{32}^{(1)} & p_{33}^{(1)}
\end{array}\right)=\frac{1}{20} \sum_{i j k} n_{i j k} E_{i j k} \\
= & 0.65\left(\begin{array}{lll}
1 & 0 & 0 \\
0 & 1 & 0 \\
0 & 0 & 1
\end{array}\right)+0.20\left(\begin{array}{ccc}
1 & 0 & 0 \\
0 & 0 & 1 \\
0 & 1 & 0
\end{array}\right)+0.05\left(\begin{array}{lll}
0 & 1 & 0 \\
1 & 0 & 0 \\
0 & 0 & 1
\end{array}\right) \\
& +0.10\left(\begin{array}{lll}
0 & 1 & 0 \\
0 & 0 & 1 \\
1 & 0 & 0
\end{array}\right)=\left(\begin{array}{ccc}
0.85 & 0.15 & 0 \\
0.1 & 0.65 & 0.25 \\
0.05 & 0.2 & 0.75
\end{array}\right) .
\end{aligned}
$$

Similarly, examine 22-nd expert preferences (4.2). Preferences of 22-nd expert are sorted and summarized in the Table 6.

Table 6. Summarized frequencies of the 22-nd expert.

\begin{tabular}{cccccccc}
\hline & $E_{123}$ & $E_{132}$ & $E_{213}$ & $E_{231}$ & $E_{312}$ & $E_{321}$ & $\Sigma$ \\
\hline$n_{i j k}$ & 7 & 3 & 8 & 1 & 1 & 0 & 20 \\
\hline
\end{tabular}

Calculate elements of 22-nd expert preferences probability matrix:

$$
\begin{aligned}
P^{(22)}= & 0.35\left(\begin{array}{lll}
1 & 0 & 0 \\
0 & 1 & 0 \\
0 & 0 & 1
\end{array}\right)+0.15\left(\begin{array}{lll}
1 & 0 & 0 \\
0 & 0 & 1 \\
0 & 1 & 0
\end{array}\right)+0.40\left(\begin{array}{lll}
0 & 1 & 0 \\
1 & 0 & 0 \\
0 & 0 & 1
\end{array}\right) \\
& +0.05\left(\begin{array}{lll}
0 & 1 & 0 \\
0 & 0 & 1 \\
1 & 0 & 0
\end{array}\right)+0.05\left(\begin{array}{lll}
0 & 0 & 1 \\
1 & 0 & 0 \\
0 & 1 & 0
\end{array}\right)=\left(\begin{array}{ccc}
0.5 & 0.45 & 0.05 \\
0.45 & 0.35 & 0.2 \\
0.05 & 0.2 & 0.75
\end{array}\right) .
\end{aligned}
$$

Note that main diagonal element of the second row of matrix 0.35 is smaller than the other item 0.45 of this row. This could be ground to question whether the 22-nd experts estimates (4.2) are consistent with the "correct" opinion (4.3). However, in this paper we do not investigate this issue. Note that it is possible to calculate averages of some or all expert assessments. If numbers of experts or variables are large we can consider the statistical estimates of the elements of probability matrix $P$. Finally, we calculate generalized experts' preferences probability matrix. The elements of this matrix are the averages 
of the correspondent elements of 22 experts' decision matrices. The obtained generalized decision matrix is:

$$
P_{1}=\left(\begin{array}{ccc}
0.702 & 0.24 & 0.057 \\
0.257 & 0.52 & 0.227 \\
0.041 & 0.24 & 0.716
\end{array}\right)
$$

The other group of 9 criteria $y_{1}-y_{9}$ described in [4] was investigated in the same manner. As a result matrix $P_{2}$ was obtained:

$$
P_{2}=\left(\begin{array}{lll}
0.843 & 0.148 & 0.009 \\
0.143 & 0.738 & 0.119 \\
0.014 & 0.114 & 0.872
\end{array}\right)
$$

Matrix $P_{1}$ corresponds to the general opinion of "average" experts, while matrix $P_{2}$ reflects the opinion of "very good" experts. Henceforth we'll analyze expert's preferences probability matrices $\tilde{P}_{1}$ and $\tilde{P}_{2}$ which are equivalent to matrices $P_{1}$ and $P_{2}$ (distributions described by the corresponding matrices do not differ with the confidence level 0.05):

$$
\tilde{P}_{1}=\left(\begin{array}{ccc}
0.7 & 0.25 & 0.05 \\
0.25 & 0.5 & 0.25 \\
0.05 & 0.25 & 0.7
\end{array}\right), \quad \tilde{P}_{2}=\left(\begin{array}{ccc}
0.85 & 0.14 & 0.01 \\
0.14 & 0.72 & 0.14 \\
0.01 & 0.14 & 0.85
\end{array}\right)
$$

\section{Numerical experiment}

Suppose, that $n$ experts $(n=2,3,4,5)$ evaluated 4 projects according to $m=3$ criteria $t_{i} \in\{1,2,3\}, i=1,2,3$, i.e. each criterion value is a natural number from 1 to 3 . Let's choose the following degrees of uncertainty: $\alpha=0.4$ and $\beta=0.1$. Our purpose is to compare the accuracy of evaluation of these projects by different aggregation functions - simple arithmetic and simple geometric average values; arithmetic and geometric averaging operators of triangular intuitionistic fuzzy numbers. We will analyze the case when the true criteria values are known, these values are represented in the Table 7 .

Table 7. True criteria $x_{1}, x_{2}, x_{3}$ values for 4 given projects.

\begin{tabular}{cccc}
\hline Projects & $x_{1}$ & $x_{2}$ & $x_{3}$ \\
\hline$p_{1}$ & 1 & 1 & 1 \\
$p_{2}$ & 1 & 2 & 2 \\
$p_{3}$ & 2 & 2 & 3 \\
$p_{4}$ & 3 & 3 & 3 \\
\hline
\end{tabular}

It's obvious that the true ranking order of projects is as follows: $p_{1} \prec p_{2} \prec$ $p_{3} \prec p_{4}$, since the Pareto dominance is valid: $(\forall j=1,2,3): x_{j}^{(2)} \geqslant x_{j}^{(1)}$ and $(\exists i): x_{i}^{(2)}>x_{i}^{(1)}$. We will conduct Monte Carlo experiments and compare errors in ranking alternatives while applying 4 aggregation functions. For matrix 
$\tilde{P}_{1}$ experts evaluations are randomly generated according to probability distributions given in the corresponding columns of Table 2. In the case of matrix $\tilde{P}_{2}$ probability distributions were calculated by analogy. Then arithmetic and geometric averages of estimates are calculated: for each project $(j=1,2,3,4)$ all criteria estimates of all experts were averaged by formulas:

$$
A\left(p_{j}\right)=\frac{1}{3 k} \sum_{j=1}^{k} \sum_{i=1}^{3} x_{i}^{(j)}, \quad G\left(p_{j}\right)=\sqrt[3 k]{\prod_{j=1}^{k} \prod_{i=1}^{3} x_{i}^{(j)}},
$$

here $k=2,3,4,5$ is the number of experts. Then generalized estimates in the form of triangular intuitionistic fuzzy numbers accumulating opinions of all experts (presented in the Table 1) are calculated for each criteria of each project. Next, these generalized estimates are normalized by formulas (2.2). As the result for each criterion of all projects we obtained triangular intuitionistic fuzzy numbers

$$
\tilde{A_{i j}}=\left(\left[a_{i j}^{L}, a_{i j}^{M}, a_{i j}^{U}\right],\left[b_{i j}^{L}, b_{i j}^{M}, b_{i j}^{U}\right]\right), \quad i=1,2,3, j=1,2,3,4 .
$$

These numbers are aggregated as arithmetic averaging operator of triangular intuitionistic fuzzy numbers [17]:

$$
\begin{aligned}
& f_{\omega}\left(\tilde{A_{1}}, \tilde{A}_{2}, \ldots, \tilde{A_{n}}\right)=\sum_{i=1}^{n} \omega_{i} \tilde{A}_{i} \\
&=\left(\left[1-\prod_{i=1}^{n}\left(1-a_{i}^{L}\right)^{w_{i}}, 1-\prod_{i=1}^{n}\left(1-a_{i}^{M}\right)^{w_{i}}, 1-\prod_{i=1}^{n}\left(1-a_{i}^{U}\right)^{w_{i}}\right],\right. \\
& {\left.\left[\prod_{i=1}^{n}\left(b_{i}^{L}\right)^{w_{i}}, \prod_{i=1}^{n}\left(b_{i}^{M}\right)^{w_{i}}, \prod_{i=1}^{n}\left(b_{i}^{U}\right)^{w_{i}}\right]\right) }
\end{aligned}
$$

and geometric averaging operator of triangular intuitionistic fuzzy numbers [17]:

$$
\begin{aligned}
& g_{\omega}\left(\tilde{A}_{1}, \tilde{A}_{2}, \ldots, \tilde{A}_{n}\right)=\prod_{i=1}^{n}\left(\tilde{A}_{i}\right)^{\omega_{i}} \\
&=\left(\left[\prod_{i=1}^{n}\left(a_{i}^{L}\right)^{w_{i}}, \prod_{i=1}^{n}\left(a_{i}^{M}\right)^{w_{i}}, \prod_{i=1}^{n}\left(a_{i}^{U}\right)^{w_{i}}\right]\right. \\
& {\left.\left[1-\prod_{i=1}^{n}\left(1-a_{i}^{L}\right)^{w_{i}}, 1-\prod_{i=1}^{n}\left(1-a_{i}^{M}\right)^{w_{i}}, 1-\prod_{i=1}^{n}\left(1-a_{i}^{U}\right)^{w_{i}}\right]\right), }
\end{aligned}
$$

here $n=3, \omega_{i}=\frac{1}{3}, i=1,2,3$. Then values of score function for triangular intuitionistic fuzzy numbers were calculated.

Definition. Suppose that $\tilde{A}=\left(\left[a^{L}, a^{M}, a^{U}\right],\left[b^{L}, b^{M}, b^{U}\right]\right)$, is a triangular intuitionistic fuzzy number. Then $S_{\tilde{A}} \in[-1 ; 1]$ is called a score function of $\tilde{A}$ :

$$
S(\tilde{A})=\left(a^{L}-b^{L}+a^{M}-b^{M}+a^{U}-b^{U}\right) / 3 .
$$

Then projects are ranked according to the values of score function. The bigger is the value of score function the better is the respective project. 
Table 8. Average values and standard deviations of error probabilities in ranking alternatives for different aggregation functions. Matrix $\tilde{P}_{1}$ ("average" experts).

\begin{tabular}{|c|c|c|c|c|c|c|c|c|}
\hline \multirow{2}{*}{ Experts } & \multicolumn{4}{|c|}{ Average values } & \multicolumn{4}{|c|}{ Standard deviations } \\
\hline & $g_{\omega}$ & $f_{\omega}$ & $A$ & $G$ & $g_{\omega}$ & $f_{\omega}$ & $A$ & $G$ \\
\hline 2 & 0.391 & 0.399 & 0.471 & 0.472 & 0.014 & 0.012 & 0.014 & 0.014 \\
\hline 3 & 0.271 & 0.253 & 0.299 & 0.300 & 0.015 & 0.014 & 0.016 & 0.016 \\
\hline 4 & 0.186 & 0.162 & 0.194 & 0.194 & 0.013 & 0.011 & 0.012 & 0.012 \\
\hline 5 & 0.134 & 0.111 & 0.128 & 0.128 & 0.011 & 0.010 & 0.011 & 0.011 \\
\hline
\end{tabular}

Table 9. Average values and standard deviations of error probabilities in ranking alternatives for different aggregation functions. Matrix $\tilde{P}_{2}$ ("very good" experts).

\begin{tabular}{|c|c|c|c|c|c|c|c|c|}
\hline \multirow{2}{*}{ Experts } & \multicolumn{4}{|c|}{ Average values } & \multicolumn{4}{|c|}{ Standard deviations } \\
\hline & $g_{\omega}$ & $f_{\omega}$ & $A$ & $G$ & $g_{\omega}$ & $f_{\omega}$ & $A$ & $G$ \\
\hline 2 & 0.098 & 0.104 & 0.129 & 0.129 & 0.010 & 0.011 & 0.012 & 0.012 \\
\hline 3 & 0.041 & 0.036 & 0.043 & 0.043 & 0.007 & 0.007 & 0.0072 & 0.0072 \\
\hline 4 & 0.017 & 0.012 & 0.017 & 0.017 & 0.004 & 0.003 & 0.0041 & 0.0042 \\
\hline 5 & 0.014 & 0.011 & 0.012 & 0.012 & 0.004 & 0.003 & 0.0038 & 0.0038 \\
\hline
\end{tabular}

500 simulations were repeated 50 times and the average probabilities of incorrect rankings of alternatives were calculated. The results of numerical experiments for matrix $\tilde{P}_{1}$ are presented in the Table 8. Minimum values of error probabilities averages and standard deviations are marked with the bold font. Corresponding results for matrix $\tilde{P}_{2}$ are presented in the Table 9 .

For both matrices the smallest average error probabilities values for 3,4 and 5 experts were obtained for arithmetic averaging operator of triangular intuitionistic fuzzy numbers $f_{\omega}$. In the case of 2 experts the smallest average error probability was obtained for geometric averaging operator of triangular intuitionistic fuzzy numbers $g_{\omega}$. For matrix $\tilde{P}_{2}$ the least standard deviation values were also obtained for the operator $f_{\omega}$ for 3,4 and 5 experts and for the operator $g_{\omega}$ in the case of 2 experts. For matrix $\tilde{P}_{1}$ standard deviations were at least for the operator $f_{\omega}$ for all numbers of experts. The dependence of average error probability on the number of experts for different aggregation operators for matrix $\tilde{P}_{1}$ is depicted in Figure 1 .

Next we searched values of parameters $\alpha$ and $\beta$ for which average errors probabilities are at least. Primarily we fixed value $\beta=0.05$ and varied $\alpha$. Least values for average error probabilities were obtained for $\alpha=0.4$ for the both operators $f_{\omega}$ and $g_{\omega}$. Then we fixed $\alpha=0.4$ and varied $\beta$. Least errors were achieved for $\beta=0.05$ with operator $g_{\omega}$ and $\beta=0.03$ with operator $f_{\omega}$. Results for matrix $\tilde{P}_{1}$ are presented in Table 10.

\section{Conclusions}

Theory of Intuitionistic Fuzzy Sets (IFS) summarizes the Fuzzy Sets (FS) concept taking into account the fact that the dependence and independence of certain elements to the fuzzy sets have a degree of uncertainty. This expands the possibilities of using the IFS as a modelling tool. In this article separate 


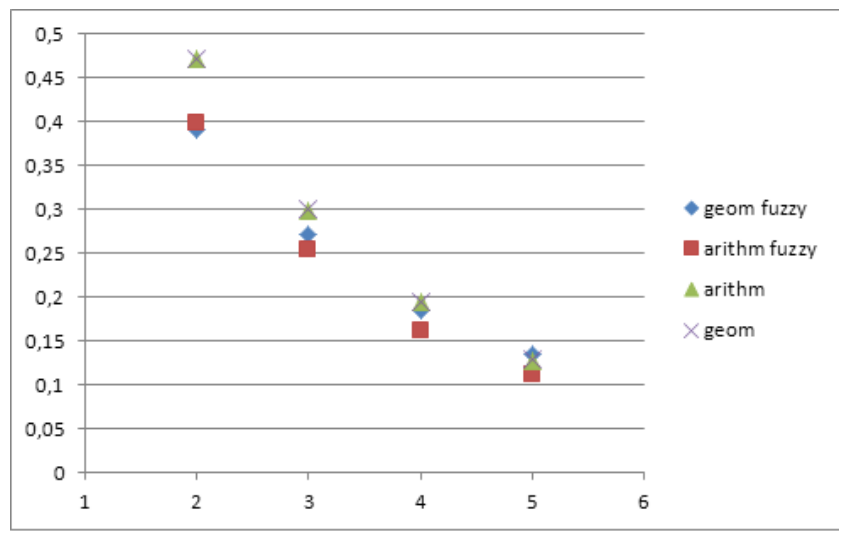

Figure 1. Average error probabilities for 2-5 experts and different aggregation operators. Matrix $\tilde{P}_{1}$.

Table 10. Average values of error probabilities dependency on $\alpha$ and $\beta$. Matrix $\tilde{P}_{1}$.

\begin{tabular}{|c|c|c|c|c|c|}
\hline \multicolumn{3}{|c|}{$\beta=0.05$} & \multicolumn{3}{|c|}{$\alpha=0.4$} \\
\hline$\alpha$ & $g_{\omega}$ & $f_{\omega}$ & $\beta$ & $g_{\omega}$ & $f_{\omega}$ \\
\hline 0.01 & 0.20914 & 0.49012 & 0.01 & 0.13962 & 0.1069 \\
\hline 0.02 & 0.19884 & 0.49804 & 0.02 & 0.137 & 0.10692 \\
\hline 0.05 & 0.18144 & 0.3514 & 0.03 & 0.13628 & 0.10258 \\
\hline 0.1 & 0.15456 & 0.41556 & 0.04 & 0.1359 & 0.10428 \\
\hline 0.2 & 0.1485 & 0.14646 & 0.05 & 0.13352 & 0.10432 \\
\hline 0.3 & 0.13664 & 0.1158 & 0.07 & 0.13396 & 0.10774 \\
\hline 0.4 & 0.13416 & 0.1107 & 0.08 & 0.13388 & 0.10912 \\
\hline 0.5 & 0.14238 & 0.11218 & 0.1 & 0.13676 & 0.112 \\
\hline 0.6 & 0.1453 & 0.11668 & 0.15 & 0.1377 & 0.11734 \\
\hline 0.7 & 0.16202 & 0.13104 & 0.2 & 0.14338 & 0.1303 \\
\hline 0.8 & 0.17074 & 0.14384 & 0.25 & 0.1474 & 0.1411 \\
\hline 0.9 & 0.18806 & 0.15982 & 0.3 & 0.15992 & 0.16014 \\
\hline 1.0 & 0.20372 & 0.17852 & 0.4 & 0.18836 & 0.20732 \\
\hline
\end{tabular}

case of IFS - intuitionistic fuzzy triangles - applied for expert estimates averaging. Monte Carlo numerical experiments were performed with hypothetical expert assessments reliability matrix. Experimental results showed that the proposed algorithms based on intuitionistic fuzzy triangles are more reliable compared to traditional statistical averages when number of experts is limited $(2,3,4,5)$. In practice a small number of experts encountered more frequently than large number of experts. For this reason large number of experts had not been examined in greater depth. By the way, we can see from the Table 8 and Table 9 that when increasing number of experts in all cases error probabilities decrease. When the number of experts is large it is likely that the law of large numbers have influence on the results and fuzzy aggregation functions also have the advantage comparing with simple averages. So, the superiority of proposed method based on intuitionistic fuzzy triangles leads to a lower error probability comparing with traditional statistical averages. 
Moreover, $\alpha$ and $\beta$ values minimizing error probability, were obtained.The article also shows how to obtain expert assessments reliability matrices of real data.

\section{References}

[1] Y. Ahmad, S. Husain and A. Ali Khan. Advancements in multi-criteria decision making based on interval-valued intuitionistic fuzzy set. Int. J. Eng. Res. Dev., 6(10):71-76, 2013.

[2] K. Atanassov. Intuitionistic Fuzzy Sets. Theory and Applications. Springer Physica-Verlag, 1999.

[3] R. Bellman and L. Zadeh. Decision making in a fuzzy environment. Management Sci., 17(4):141-164, 1970. http://dx.doi.org/10.1287/mnsc.17.4.B141.

[4] S. Dadelo, A. Krylovas, N. Kosareva, E. K. Zavadskas and R. Dadeliene. Algorithm of maximizing the set of common solutions for several mcdm problems and it's application for security personnel scheduling. Int. J. Comput. Commun. Control, 9(2):151-159, 2014. http://dx.doi.org/10.15837/ijccc.2014.2.1015.

[5] A. Nagoor Gani, N. Sritharan and C. Arun Kumar. Weighted average rating (WAR) method for solving group decision making problem using an intuitionistic trapezoidal fuzzy hybrid aggregation (ITFHA) operator. Int. J. Pure Appl. Sci. Technol., 6(1):54-61, 2011.

[6] Y. He, H. Chen, L. Zhou, B. Han, Q. Zhao and J. Liu. Generalized intuitionistic fuzzy geometric interaction operators and their application to decision making. Expert Systems Appl., 41(5):2484-2495, 2014. http://dx.doi.org/10.1016/j.eswa.2013.09.048.

[7] S. Husain, Y. Ahmad and M. Afshar Alam. A study on the role of intuitionistic fuzzy set in decision making problems. Int. J. Comput. Appl., 48(19):35-41, 2012 .

[8] S. Jose and S. Kuriakose. Note on multiattribute decision making in intuitionistic fuzzy context. Notes on Intuitionistic Fuzzy Sets, 19(1):48-53, 2013.

[9] N. Kosareva and A. Krylovas. Comparison of accuracy in ranking alternatives performing generalized fuzzy average functions. Technological and Economic Development of Economy, 19(1):162-187, 2013. http://dx.doi.org/10.3846/20294913.2012.763072.

[10] P. Liu. Some Hamacher aggregation operators based on the interval-valued intuitionistic fuzzy numbers and their application to group decision making. IEEE Trans. Fuzzy Systems, 22(1):83-97, 2014.

http://dx.doi.org/10.1109/TFUZZ.2013.2248736.

[11] P. Liu, Z. Liu and X. Zhang. Some intuitionistic uncertain linguistic Heronian mean operators and their application to group decision making. Appl. Math. Comput., 230(1):570-586, 2014. http://dx.doi.org/10.1016/j.amc.2013.12.133.

[12] Y. Luo, X. Li, Y. Yang and Z. Liu. Some models for multiple attribute decision making with intuitionistic fuzzy information and uncertain weights. Int. J. Comput. Sci. Issues, 10(1):262-266, 2013.

[13] J. Wang, R. Nie, H. Zhang and X. Chen. Intuitionistic fuzzy multi-criteria decision-making method based on evidential reasoning. Appl. Soft Comput., 13(4):1823-1831, 2013. http://dx.doi.org/10.1016/j.asoc.2012.12.019. 
[14] G. Wei, H. Wang, X. Zhao and R. Lin. Hesitant triangular fuzzy information aggregation in multiple attribute decision making. J. Intell. Fuzzy Systems, 26(3):1201-1209, 2014.

[15] D. Yu. Intuitionistic trapezoidal fuzzy information aggregation methods and their applications to teaching quality evaluation. J. Inf. Comput. Sci., 10(6):1861-1869, 2013. http://dx.doi.org/10.12733/jics20102199.

[16] S. Zeng, T. Baležentis, J. Chen and G. Luo. A projection method for multiple attribute group decision making with intuitionistic fuzzy information. Informatica, 24(3):485-503, 2013.

[17] X. Zhang and P. Liu. Method for aggregating triangular fuzzy intuitionistic fuzzy information and its application to decision making. Technological and Economic Development of Economy, 16(2):280-290, 2010.

http://dx.doi.org/10.3846/tede.2010.18. 